\title{
Multiple idiopathic cervical root resorption most frequently seen in younger females
}

\author{
What are the history, clinical findings and radiographic appearance of multiple \\ idiopathic cervical root resorption?
}

\begin{abstract}
Liang H, Burkes EJ, Frederiksen NL. Multiple idiopathic cervical root resorption: systematic review and report of four cases. Dentomaxillofac Radiol 2003; 32: 150-155
\end{abstract}

Data sources Medline and references of included studies were used to source articles.

Study selection Selected articles were case reports or reviews of patients who had multiple root resorption with no systemic or local factors that may have contributed to root resorption, the resorption originating at the cemento-enamel junction of teeth and involving more than three teeth in the dentition.

Data extraction and synthesis Clinical, histological and historical findings are summarised for all identified patients.

Results Multiple idiopathic cervical root resorption (MICRR) was an incidental finding on routine clinical and radiographic examination. There appeared to be no correlation between this type of resorption and any medical or dental finding. Radiographically, MICRR was found to begin at the cemento-enamel junction and then either progress to involve the entire cervical region or, at some point, spontaneously arrest. Those cases that progressed to involve the entire cervical region required extraction. The number of teeth that demonstrated this condition ranged from five up to 24 per patient. More teeth became involved as the condition was followed in time. There was no detectable frequency of occurrence for any particular dental region or tooth among the involved teeth. Of a total of 18 patients, 13 were females whose ages ranged from 7 to 68 years of age. Ten of the 18 patients were Caucasian.

Conclusions Multiple idiopathic cervical root resorption was found most frequently associated with younger females. This condition appeared to be of unknown aetiology and uncertain natural history.

\section{Commentary}

This report follows a well-established approach to the systematic review, with particular regard for clinical and radiological features of a particular lesion. The authors are American diagnostic clinicians (two are oral and maxillofacial radiologists).

In addition to 10 reported cases of MICRR that fulfilled the selection criteria, the authors presented four cases. Seven of the 10 reports were recovered from Medline and three others were discovered upon review of the citation lists of identified reports.

Only three of the 10 reported more than one case. A problem with single case reports is that it is often difficult to determine their frequency as the period over which the search for such cases is often not reported. Handsearching does not appear to have been done, not an uncommon occurrence in dental systematic reviews ${ }^{1}$ even though, despite its time-consuming and expensive nature, it does enhance the validity of the review.

The apparent great difference in mean age between the sexes is not significant $(t, 1.26)$. The authors revealed that MICRR is an asymptomatic (therefore, occult) progressive condition. They clearly established the clinical differences between MICRR and root caries. The value of this report is that it will prompt the clinician to consider it when presented with root caries on many teeth. This is important as the implied conclusion of this paper is that it is likely that a proportion of MICRR may have been misdiagnosed and treated as root caries. Diagnosis of more cases may make more certain whether such features as speed and progression of the disease, and hormonal abnormalities (whether they have a role to play and, if so, what that role is). This report is a valuable starting point for further work on MICRR.

\section{David MacDonald-Jankowski}

Faculty of Dentistry, University of British Columbia, Vancouver, British Columbia, Canada

1. Glenny AM, Esposito M, Coulthard P, Worthington HV. The assessment of systematic reviews in dentistry. Eur J Oral Sci 2003; 111:85-92.

Evidence-Based Dentistry (2005) 6, 20.

doi:10.1038/sj.ebd.6400308 